\title{
Influence of suspension viscosity on Brownian relaxation of filler particles
}

\author{
Rick Dannert $^{1} \cdot$ H. Henning Winter ${ }^{2} \cdot$ Roland Sanctuary $^{1} \cdot$ Jörg Baller $^{1}$
}

Received: 2 November 2016 /Revised: 20 April 2017 / Accepted: 15 May 2017 /Published online: 7 June 2017

(C) The Author(s) 2017. This article is an open access publication

\begin{abstract}
Brownian relaxation caused by Brownian movement of particles in suspensions can macroscopically be probed by small-amplitude oscillatory shear experiments. Phenomenological considerations suggest a direct proportionality between suspension viscosity and Brownian relaxation times. To verify this relation experimentally, a set of nanocomposite suspensions with viscosities varying over five decades is presented. The suspensions are chosen in a way to ensure that particle-particle interactions and average particle-particle distances are identical so that they can be used as a model system to study the mere influence of suspension viscosity on Brownian relaxation. The suggested linear relationship between suspension viscosity and Brownian relaxation time can be confirmed. Moreover, a verification of a recently introduced characteristic timescale for Brownian relaxation is presented.
\end{abstract}

Keywords BSW spectrum · Nanocomposite $\cdot$ Brownian relaxation $\cdot$ SAOS $\cdot$ Glass transition $\cdot$ Master curve

\section{Introduction}

In colloidal suspensions, spatial and temporal fluctuations of the particle density due to thermal agitation

Jörg Baller

joerg.baller@uni.lu

1 Physics and Materials Science Research Unit, Laboratory for the Physics of Advanced Materials, University of Luxembourg, 162A Avenue de la Faïencerie, L-1511 Luxembourg, Luxembourg

2 Chemical Engineering Department and Polymer Science and Engineering Department, University of Massachusetts Amherst, Amherst, MA 01003-3110, USA lead to macroscopic fluctuations of the so-called Brownian stress. Brownian stress and its relaxation (referred to as Brownian relaxation) can be probed by oscillatory shear experiments respecting linear response conditions. Shikata and Pearson (1994) realized oscillatory shear measurements on ethylene glycol-glycerol mixtures, filled with silica submicron particles (volume concentrations, $x_{\mathrm{V}} \geq 0.32$ ). The samples investigated by van der Werff et al. (1989) consisted of sterically stabilized silica particles dispersed in cyclohexane with volume fractions $\left(x_{\mathrm{V}}\right)$ varying between 0.3 and 0.6. Watanabe et al. (1997) carried out oscillatory shear measurements on a $50 \mathrm{wt} \%$ suspension of spherical silica nanoparticles in a super-cooled ethylene glycol-glycerol mixture. In all cases, the fillers were considered as hardsphere particles and the Brownian relaxation time was shown to depend in the same way on the filler concentration as the Peclet time defined as the characteristic time needed for a suspended spherical particle to diffuse along a distance equal to its own radius.

In the present contribution, diglycidyl ether of bisphenol A (DGEBA) is used as a suspension matrix. DGEBA is a low molecular weight $\left(374 \mathrm{~g} \mathrm{~mol}^{-1}\right)$ glass former which is not known to show any relaxation process slower than the structural $\alpha$-relaxation in rheological measurements (White 2013). Due to the small molecular weight of the DGEBA molecules, entanglements leading to a rubbery plateau in rheological measurements are not expected. Wideband dielectric spectroscopy (Capaccioli et al. 1998; Corezzi et al. 2002) also does not give a hint to slow relaxation processes in pure DGEBA. The situation changes when adding nanoscaled filler particles to the DGEBA matrix: In previous works, Dannert et al. $(2015,2014)$ have published data on a low-frequency relaxation process observed in 
colloidal suspensions formed by DGEBA and spherical silica nanoparticles. In contrast to other matrix relaxation processes found in DGEBA such as $\alpha$-relaxation, $\beta$-relaxation and the strong $\gamma$-relaxation, the newly reported low-frequency process does not show an independent temperature dependency. It shows the same temperature dependency as the structural relaxation process ( $\alpha$-process), which leads to the conclusion that it is related to the matrix viscosity. The most prominent experimental feature reported in Dannert et al. (2014) is the scaling of the relaxation times of the low-frequency process with the Peclet time at sufficiently high filler fractions. In line with the interpretation suggested in Shikata and Pearson (1994), van der Werff et al. (1989) and Watanabe et al. (1997), this low-frequency event was described as a Brownian relaxation. In contrast to the works cited above, it has been shown (Dannert et al. 2015) that the Peclet time is not able to predict Brownian relaxation times in dilute suspensions. To adequately describe the evolution of the relaxation time as a function of $x_{\mathrm{V}}$, a modified Peclet time has been introduced by substituting the particle radius by the average interparticle distance. The use of the mean particle distance as a characteristic length scale has been motivated by the fact that interactions between particles (scaling with the particle distance) are a prerequisite for Brownian relaxation.

Regardless of the used length scale, in case that the StokesEinstein relation is valid, both time scales (Peclet time and modified Peclet time) predict a linear scaling of Brownian relaxation times with the suspension viscosity (Banchio et al. 1999; Sohn and Rajagopalan 2004). However, to our very best knowledge, in the literature, no experimental data are available proving the linear dependency between Brownian relaxation times and suspension viscosity. Therefore, in the present paper, we will first demonstrate that we could produce a series of suspensions, which exhibit viscosities ranging over several orders of magnitude, while exactly showing the same complex rheological behaviour. This series of suspensions is then used as a model system to study the relation between suspension viscosity and Brownian relaxation times.

\section{Materials and experimental setup}

\section{Materials}

\section{DGEBA/BPA matrices}

DGEBA (DER distilled) with a molecular weight $M_{\mathrm{n}}=374$ $\mathrm{g} \mathrm{mol}^{-1}$ purchased from Nanoresins AG (Geesthacht, Germany) has been used as a base material for all investigated samples. DGEBA is an almost ideal glass former with very low tendency to crystallize and with a glass transition temperature of about $257 \mathrm{~K}$ (Baller et al. 2009). The degree of polymerization of DGEBA can be varied by curing DGEBA with bisphenol A (BPA). During the curing mechanism, the oxirane rings of the DGEBA molecules are able to react with the hydroxyl groups of BPA leading to an almost linear polymerization. In order to create matrices with different degrees of polymerization, the base material DGEBA has been cured with different mass ratios: $r_{\mathrm{BPA}}=m_{\mathrm{BPA}} / m_{\mathrm{DGEBA}}$ of BPA. For this study, matrices with $r_{\mathrm{BPA}}=0.3,0.5$ and 0.7 have been produced. BPA was purchased from Sigma-Aldrich (purity $>99 \%$, melting temperature about $431 \mathrm{~K}$ ). The following curing method was applied: In the first step, a planetary mixer was used to homogeneously disperse the appropriated amount of BPA (crystalline at room temperature) inside distilled DGEBA. Next, the samples were kept at $393 \mathrm{~K}$ until all of the BPA had reacted with DGEBA. This temperature was chosen to avoid branching reactions between DGEBA and BPA. Branching reactions are indeed reported to occur at temperatures higher than $423 \mathrm{~K}$ (Smith and Ishida 1994). For each mass ratio $\left(r_{\mathrm{BPA}}\right)$, the relative weight-average molecular weights of the DGEBA/BPA system

$M_{\mathrm{W}, r}=\frac{M_{\mathrm{W}}(\text { DGEBA } / \mathrm{BPA})}{M_{\mathrm{W}}(\text { DGEBA })}$

have been determined by gel permeation chromatography. The respective values are given in Table 1.

\section{DGEBA/SiO ${ }_{2}$ and $\mathrm{DGEBA} / \mathrm{BPA} / \mathrm{SiO}_{2}$ suspensions}

In this work, the DGEBA/ $/ \mathrm{SiO}_{2}$ suspension with a volume concentration, $x_{\mathrm{V}}=0.05$, of nanoparticles was used. It was obtained by adequately diluting DGEBA filled with $25 \mathrm{vol} \%$ of silica nanoparticles (Nanopox A410, Nanoresins AG, Geesthacht, Germany) with distilled DGEBA. The silica particles have a quasi-spherical shape with an average radius of about $12.5 \mathrm{~nm}$ including an ultra-thin stabilization layer (Baller et al. 2009; Sanctuary et al. 2006). DGEBA/BPA/ $\mathrm{SiO}_{2}$ suspensions with a concentration of $x_{\mathrm{V}}=0.05$ of silica

Table 1 Relative weight-average molecular weights $\left(M_{\mathrm{W}, r}\right)$ of the DGEBA/BPA system for the different mass ratios $\left(r_{\mathrm{BPA}}=m_{\mathrm{BPA}} /\right.$ $\left.m_{\text {DGEBA }}\right)$. The $M_{\mathrm{W}, r}$ values have been obtained by gel permeation chromatography (GPC)

\begin{tabular}{ll}
\hline$r_{\mathrm{BPA}}$ & $M_{\mathrm{W}, r}$ \\
\hline 0 & 1 \\
0.3 & 1.57 \\
0.5 & 1.67 \\
0.7 & 1.9 \\
\hline
\end{tabular}


nanoparticles were obtained by curing DGEBA/SiO $\mathrm{S}_{2}$ suspensions with BPA using the procedure described in the previous section. Hence, the silica nanoparticles are already well dispersed in one of the reactants before polyaddition takes place (Baller et al. 2009).

\section{Experimental methods}

Dynamic rheological measurements were performed with an Anton Paar rheometer (MCR 302) using parallel-plate geometry. To avoid compliance effects, the diameter of the plates $(8,25$ or $50 \mathrm{~mm}$ ) was chosen depending on the dynamic stiffness of the samples. For the measurement of the highest shear moduli, the plates with the smallest diameter were chosen and, for the measurement of the lowest moduli, the plates with the largest radius. Isothermal frequency sweeps were carried out at different temperatures $(T)$ in order to determine the complex shear modulus: $G^{*}(\omega)=G^{\prime}(\omega)+i G^{\prime \prime}(\omega)$, where $G^{\prime}(\omega)$ and $G^{\prime \prime}(\omega)$ represent the storage modulus and the loss shear modulus, respectively. The temperature ranges for the rheological characterization of the different samples are listed in Table 2. The frequency window exploited for the measurements extended from $\omega=0.314 \mathrm{rad} \mathrm{s}^{-1}$ to $\omega=62.8 \mathrm{rad} \mathrm{s}^{-1}$. Measurements were carried out in constant strain or constant stress mode to obtain the best accuracy possible. A linear response of the samples was verified for each frequency $(\omega)$ and every temperature $(T)$. Using the IRIS program (Winter and Mours 2006), the frequency-temperature equivalence principle was exploited to combine the measured shear data to master curves at the reference temperature $\left(T_{\text {ref }}=273 \mathrm{~K}\right)$.

Since the experimental results, which are mainly discussed in the following, are obtained from rheological measurements at very low frequencies, the reliability of data when the phase angle approaches $90^{\circ}$ needs to be discussed. Figure 1 shows

Table 2 Temperature ranges within which the isothermal frequency sweeps where carried out on DGEBA/BPA samples and DGEBA/BPA/ $\mathrm{SiO}_{2}$ suspensions

\begin{tabular}{lll}
\hline Sample & $T_{\min }(\mathrm{K})$ & $T_{\max }(\mathrm{K})$ \\
\hline$r_{\mathrm{BPA}}=0$ & 255 & 273 \\
$r_{\mathrm{BPA}}=0$ with nanoparticles & 259 & 303 \\
$r_{\mathrm{BPA}}=0.3$ & 263 & 278 \\
$r_{\mathrm{BPA}}=0.3$ with nanoparticles & 263 & 313 \\
$r_{\mathrm{BPA}}=0.5$ & 268 & 281 \\
$r_{\mathrm{BPA}}=0.5$ with nanoparticles & 263 & 323 \\
$r_{\mathrm{BPA}}=0.7$ & 273 & 288 \\
$r_{\mathrm{BPA}}=0.7$ with nanoparticles & 273 & 343 \\
\hline
\end{tabular}

the master curves $\left(\delta\left(a_{T} \omega\right)\right)$ of the loss angle $\left(\delta=\tan ^{-1}\left(G^{\prime \prime} / G^{\prime}\right)\right)$ obtained for DGEBA $/ \mathrm{SiO}_{2}\left(r_{\mathrm{BPA}}=0\right)$ suspensions with volume concentrations, $x_{V}=0,0.054,0.11,0.18$ and 0.25 (from the measurements published in Dannert et al. (2014)).

1. As can be seen in Fig. 1, the phase angle $(\delta)$ obtained for the neat matrix $\left(x_{\mathrm{V}}=0\right)$ approaches $90^{\circ}$ at low frequencies. The cut-off of data at low frequencies is due to technical reasons: As a matter of fact, the phase angle starts fluctuating around $90^{\circ}$ when $G^{\prime \prime}$ starts exceeding $G^{\prime}$ by several orders of magnitude. The software of the Anton Paar rheometer is conceived to ignore any phase angle value larger than $90^{\circ}$ (for an obvious physical reason). This leads to average values of the phase angle, which are too low, and to storage shear modulus values, which are too high compared with those expected under terminal regime conditions.

2. The phase angle of the investigated suspensions shows a more complex behaviour at low frequencies compared to the neat matrix: Irrespective of the filler content, $\delta$ exhibits a local minimum. Common to all suspensions is the fact that, independent of the frequency, the measured phase angle lies well below $90^{\circ}$. Thus, it can be concluded that the observed low-frequency behaviour of the filled systems is clearly not stemming from measurement artefacts. The question whether the neat matrix would exhibit a similar effect if it would be possible to extend the measurement to even lower frequencies cannot be answered here. The issue will be discussed at full

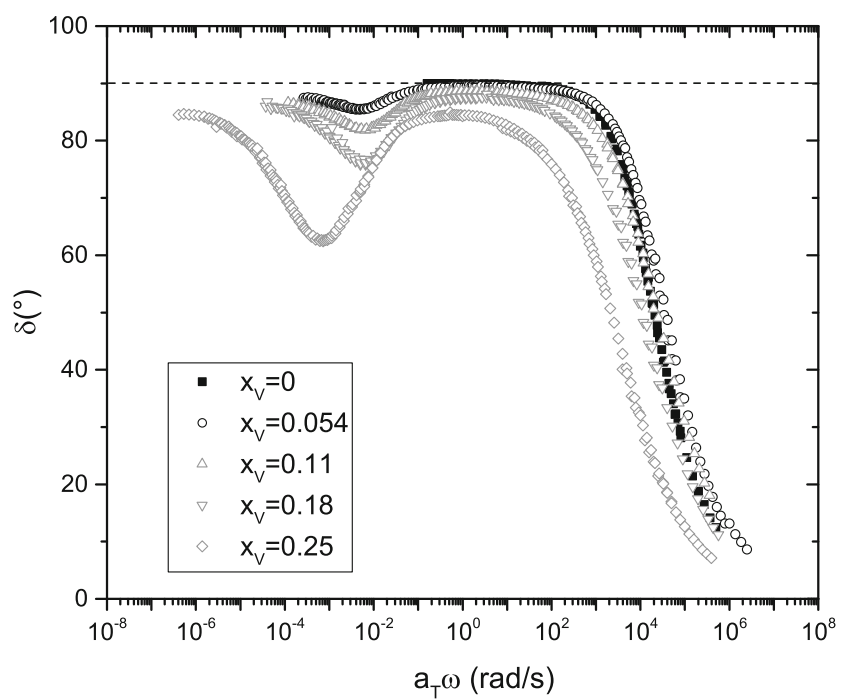

Fig. 1 Master curves $\left(\delta\left(a_{T} \omega\right)\right)$ of the loss angle of various DGEBA/SiO suspensions with volume concentration $\left(x_{\mathrm{V}}\right)$ at the reference temperature $\left(T_{\text {ref }}=273 \mathrm{~K}\right)$ taken from the measurements published in Dannert et al. (2014). The horizontal dashed line corresponding to $\delta=90^{\circ}$ is a guideline for the eyes 
length in the "Impact of nanoparticles on the rheological behaviour of DGEBA/BPA matrices" section.

\section{Results and discussion}

\section{Rheological characterization of DGEBA/BPA matrices}

Figure 2 presents double-logarithmic plots of the master curves $G^{\prime}\left(a_{T} \omega\right)$ and $G^{\prime \prime}\left(a_{T} \omega\right)$ obtained for DGEBA/BPA matrices with different mass ratios $\left(r_{\mathrm{BPA}}\right)$ of cured BPA at the reference temperature $\left(T_{\text {ref }}=273 \mathrm{~K}\right)$.

To construct the master curves, the temperature-frequency equivalence principle has been exploited. The latter allows superposing the storage and loss shear data measured at different temperatures by horizontal and modulus shifts. However, for the master curves shown in Fig. 2, no modulus shifts were necessary. This is illustrated by the van GurpPalmen plot (Van Gurp and Palmen 1998) in Fig. 3: For each sample, the loss angle $(\delta)$ as a function of $\left|G^{*}\right|$ shows a continuous evolution.

In each sample, the dynamical glass transition occurs when the loss shear modulus $\left(G^{\prime \prime}\left(a_{T} w\right)\right)$ reaches a maximum. The important shift of the dynamic glass transition towards lower frequencies with increasing BPA content represents the most important feature depicted by Fig. 2. When increasing the $\mathrm{BPA}$ concentration from $r_{\mathrm{BPA}}=0$ to $r_{\mathrm{BPA}}=0.7$, the frequency at which the glass transition occurs decreases by more than five decades (see also Table 3). The pronounced shift of the dynamic glass transition is expected to go along with a steep increase of the glass transition temperature. According to the Fox-Flory equation (Fox and Flory 1950), the change of the glass transition temperature is proportional to the inverse of the molecular weight. Thus, for a low molecular weight glass

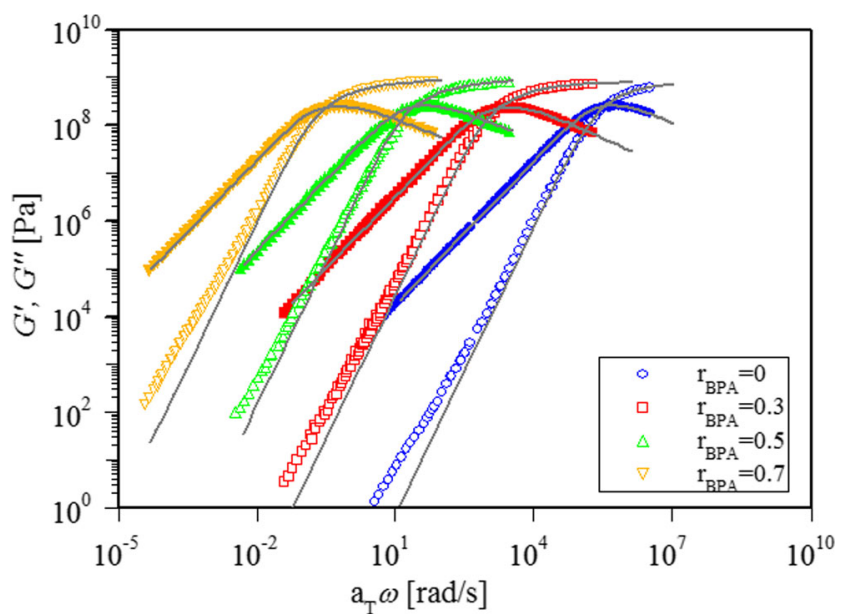

Fig. 2 Master curves $G^{\prime}\left(a_{T} \omega\right)$ (open symbols) and $G^{\prime \prime}\left(a_{T} \omega\right)$ (closed symbols) for polymerized DGEBA matrices cured with different mass ratios $\left(r_{\mathrm{BPA}}\right)$ of BPA. The solid lines correspond to $G^{\prime}(\omega)$ and $G^{\prime \prime}(\omega)$ calculated using the BSW model (Eqs. (2) and (3)). Reference temperature: $T_{\text {ref }}=273 \mathrm{~K}$ former as DGEBA, the growth of $T_{\mathrm{g}}$ is expected to be especially pronounced when its molecular weight slightly increases by the addition of BPA.

Apart from the shift of the dynamic glass transition to lower frequencies, the overall viscoelastic behaviour of the DGEBA/ BPA matrices seems not to be substantially affected by the polyaddition process. In Fig. 2, the solid lines correspond to the fits of the measured storage and loss moduli to the equations (Winter 2013)

$$
\begin{aligned}
& G^{\prime}(\omega)=\int_{0}^{\infty} \frac{H(\tau)}{\tau} \cdot \frac{(\omega \tau)^{2}}{1+(\omega \tau)^{2}} \cdot d \tau \\
& G^{\prime \prime}(\omega)=\int_{0}^{\infty} \frac{H(\tau)}{\tau} \cdot \frac{\omega \tau}{1+(\omega \tau)^{2}} \cdot d \tau
\end{aligned}
$$

where

$H(\tau)=H_{0}\left(\frac{\tau}{\tau_{\max }}\right)^{n} e^{-\left(\frac{\tau}{\tau_{\max }}\right)^{\beta}}$

represents the broadened power law spectrum (BSW model; Baumgaertel et al. (1990)).

While in the vicinity of the dynamic glass transition, all of the systems can be adequately described by the fits, deviations of the $G^{\prime}(\omega)$ data from the broadened BSW models are observed at small modulus values. As a matter of fact, at the lowest frequencies, the storage shear modulus $\left(G^{\prime}=\left|G^{*}\right| \cos \delta\right)$ systematically deviates to values higher as those expected from the BSW fits. This deviation results from measurement limitations (see the "DGEBA/SiO ${ }_{2}$ and DGEBA/BPA/SiO suspensions" section).

The values of the parameters $H_{0}, \tau_{\max }, \beta$ and $n$ used for the broadened BSW models are listed in Table 3. $\tau_{\max }$ represents the longest relaxation time, i.e. the characteristic time scale for

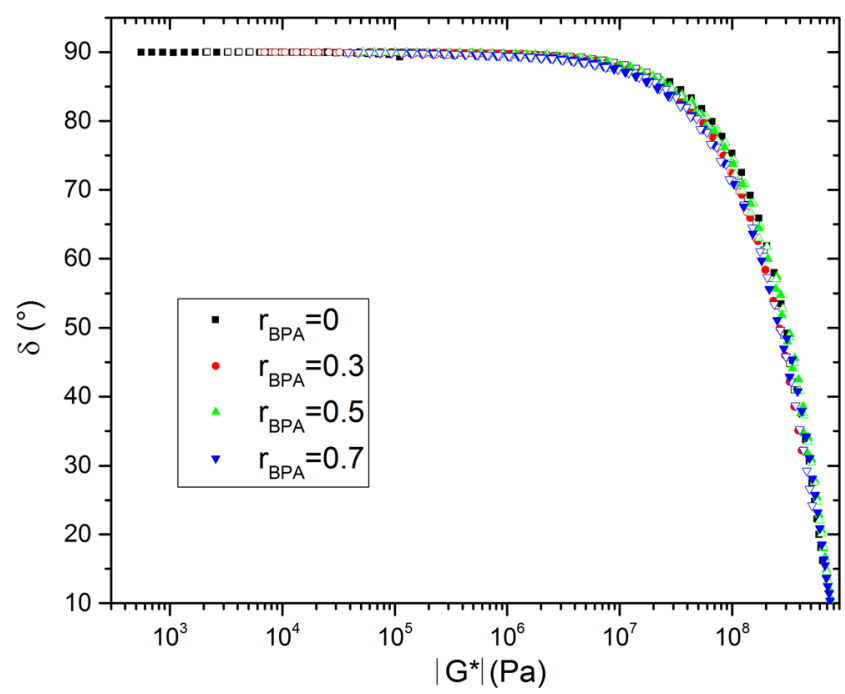

Fig. 3 Van Gurp-Palmen plots $\delta\left(\left|G^{*}\right|\right)$ for the DGEBA/BPA samples with various concentrations of BPA. The curves are synthesized from the data delivered by the isothermal frequency sweeps without any treatment 
Table 3 Parameters $H_{0}, \tau_{\max }, \beta$ and $n$ obtained by adjusting the broadened BSW model (Eqs. (2) and (3)) to the $G^{\prime}\left(a_{T} \omega\right)$ and $G^{\prime \prime}\left(a_{T} \omega\right)$ master curves in Fig. 2 together with the matrix viscosities $\left(\eta_{\mathrm{m}}\right)$ for the different mass ratios $\left(r_{\mathrm{BPA}}\right)$

\begin{tabular}{llllll}
\hline$r_{\mathrm{BPA}}(-)$ & $H_{0}\left(\times 10^{9} \mathrm{~Pa}\right)$ & $\tau_{\max }(\mathrm{s})$ & $n(-)$ & $\beta(-)$ & $\eta_{\mathrm{m}}\left(\mathrm{Pa} \mathrm{s}^{-1}\right)$ \\
\hline 0 & 0.73 & $6.02 \times 10^{-6}$ & 0.59 & 0.68 & $1.89 \times 10^{3}$ \\
0.3 & 0.57 & $2.88 \times 10^{-3}$ & 0.5 & 0.68 & $3.32 \times 10^{5}$ \\
0.5 & 0.6 & $9.5 \times 10^{-2}$ & 0.47 & 0.68 & $2.5 \times 10^{7}$ \\
0.7 & 0.55 & $7.94 \times 10^{0}$ & 0.44 & 0.68 & $9.12 \times 10^{8}$ \\
\hline
\end{tabular}

the dynamic glass transition. According to the discussion above, it increases up to 6 orders of magnitude for the matrix with the highest content of BPA. Since the relaxation time $\left(\tau_{\max }\right)$ of the structural relaxation process is usually directly related to the viscosity (see e.g. Debenedetti and Stillinger (2001)), this result is in line with the significant increase of the matrix viscosity $\left(\eta_{\mathrm{m}}\right)$ (Table 2$)$ determined from the master curves in Fig. 2 using

$\eta_{\mathrm{m}}=\left(\frac{G^{\prime \prime}\left(a_{T} \omega\right)}{a_{T} \omega}\right)_{\omega \rightarrow 0}$

The observation that all matrices exhibit a similar terminal regime-scaling behaviour (Fig. 2) can be understood by the relatively small increase of the molecular weight with a growing mass ratio of BPA. In fact, as shown in Table 1 for the highest amount of cured BPA $\left(r_{\mathrm{BPA}}=0.7\right)$, the relative weightaverage molecular weight of the DGEBA/BPA system is smaller than 2. As a consequence, the molecular weights of the DGEBA/BPA systems are too small to observe normalmode relaxations (Paluch et al. 2003) or a rubbery plateau due to entanglements (Ferry 1980).

In order to fortify this observation, the master curves of all DGEBA/BPA matrices shown in Fig. 2 have been horizontally shifted. This is illustrated in Fig. 4, and the normalization shift factors $\left(s_{\text {matrix }}\right)$ can be found in Table 4 . The curves overlap perfectly over the whole frequency range. Thus, it can be concluded that DGEBA/BPA matrices represent ideal candidates to produce suspensions with complex but similar viscoelastic properties. This is the basis for an analysis of the suspension viscosity dependency of Brownian relaxation - as announced in the "Introduction".

\section{Impact of nanoparticles on the rheological behaviour of DGEBA/BPA matrices}

Figure 5 shows the master curves $G^{\prime}\left(a_{T} \omega\right)$ and $G^{\prime \prime}\left(a_{T} \omega\right)\left(T_{\text {ref }}=\right.$ $273 \mathrm{~K}$ ) obtained for DGEBA/BPA/ $\mathrm{SiO}_{2}$ suspensions with a volume concentration, $x_{\mathrm{V}}=0.05$, of silica nanoparticles and cured with different mass ratios $\left(r_{\mathrm{BPA}}\right)$ of BPA. Similar to

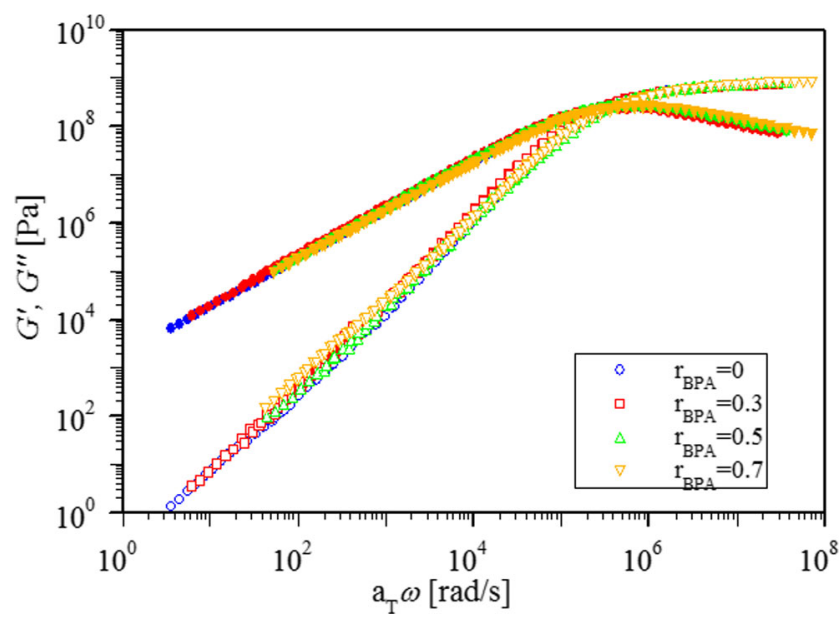

Fig. $4 G^{\prime}\left(a_{T} \omega\right)$ (open symbols) and $G^{\prime \prime}\left(a_{T} \omega\right)$ (closed symbols) from Fig. 2 shifted by horizontal factors $\left(s_{\text {matrix }}\right)$ to the master curves of the pure DGEBA matrix. The corresponding normalization shift factors $\left(s_{\text {matrix }}\right)$ can be found in Table 3

Fig. 2, the master curves have been constructed using the temperature-frequency equivalence principle. Again, no modulus shifts of the data were necessary (see the van Gurp-Palmen plots in Fig. 6). Obviously, the presence of the nanoparticles renders the rheological behaviour of the DGEBA/BPA/SiO suspensions more complex than the one observed for the neat matrices. Besides the dynamic glass transition at high frequencies, all suspensions show a plateau-like behaviour of the storage modulus at low frequencies. Moreover, the low-frequency process seems to shift to lower frequencies with increasing content $\left(r_{\mathrm{BPA}}\right)$ of the curing agent in a similar way as the dynamic glass transition.

In the same way as for the DGEBA/BPA matrices, the master curves of the DGEBA/BPA/SiO ${ }_{2}$ suspensions shown in Fig. 5 have been horizontally shifted. As illustrated in Fig. 7, the master curves of both moduli perfectly superimpose across the whole frequency range including the regions where both the structural and the low-frequency processes dominate. The corresponding normalization shift factors $\left(s_{\text {suspension }}\right)$ (see Table 4) are fairly identical to the normalization shift factors $\left(s_{\text {matrix }}\right)$ used for the DGEBA/BPA matrices.

The complex rheological behaviour of the DGEBA/BPA/ $\mathrm{SiO}_{2}$ suspensions is not affected by the curing: As a matter of fact, curing takes influence neither on the characteristics of the dynamic glass transition nor on those of the low-frequency
Table 4 Normalization shift factors $s_{\text {matrix }}$ and $s_{\text {suspension }}$ used to superimpose the master curves from Figs. 1 and 3

\begin{tabular}{lll}
\hline$r_{\mathrm{BPA}}(-)$ & $S_{\text {matrix }}(-)$ & $S_{\text {suspension }}(-)$ \\
\hline 0 & 0 & 0 \\
0.3 & 2.5 & 2.3 \\
0.5 & 4.14 & 4.23 \\
0.7 & 5.83 & 5.71 \\
\hline
\end{tabular}




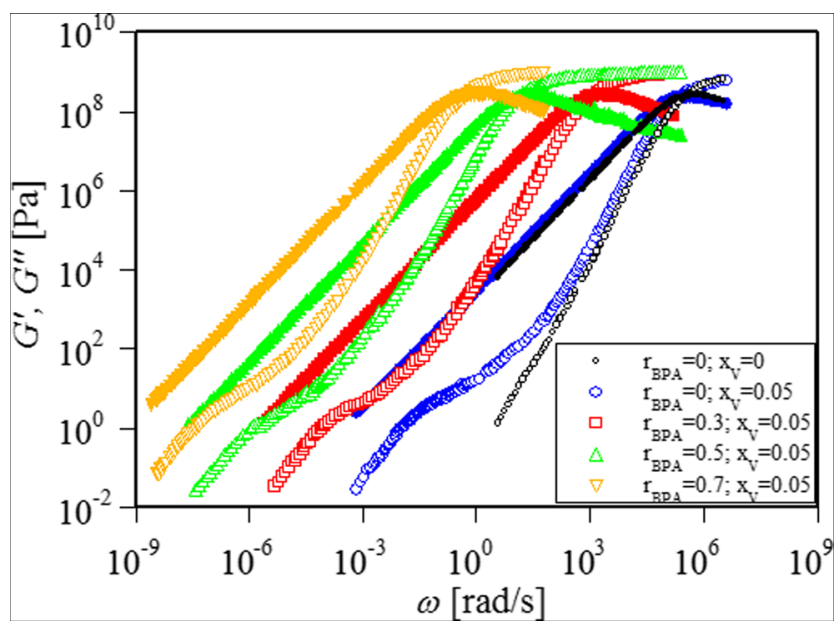

Fig. 5 Master curves $G^{\prime}\left(a_{T} \omega\right)$ (open symbols) and $G^{\prime \prime}\left(a_{T} \omega\right)$ (closed symbols) for DGEBA $\left(r_{\mathrm{BPA}}=0 ; x_{\mathrm{V}}=0\right)$ as well as for DGEBA/BPA/ $\mathrm{SiO}_{2}$ suspensions (concentration of nanoparticles $\left(x_{\mathrm{V}}=0.05\right)$, mass ratios $\left(r_{\mathrm{BPA}}\right)$ of curing agent $\mathrm{BPA}$ as indicated). Reference temperature: $T_{\text {ref }}=273 \mathrm{~K}$

process (except for the frequency shifts dependent on the BPA content).

The inset of Fig. 6 allows to evaluate the minimum of the loss angle $(\delta)$ as a function of BPA mass ratio $\left(r_{\mathrm{BPA}}\right)$ and to calculate the loss angle change $\left(\Delta \delta\left(r_{\mathrm{BPA}}\right)\right)$ induced by the nanoparticles at low frequencies (see the inset of Fig. 6 for the evaluation of $\left.\Delta \delta\left(r_{\mathrm{BPA}}\right)\right)$. From Fig. 8 , it turns out that $\Delta \delta\left(r_{\mathrm{BPA}}\right)$ does not substantially change with an increasing amount of the curing agent in the DGEBA/BPA/ $/ \mathrm{SiO}_{2}$ suspensions (with a constant filler content, $x_{\mathrm{V}}=0.05$ ). In the study of Dannert et al. (2015), the authors have investigated the lowfrequency process in DGEBA/ $/ \mathrm{SiO}_{2}$ suspensions $\left(r_{\mathrm{BPA}}=0\right)$ as

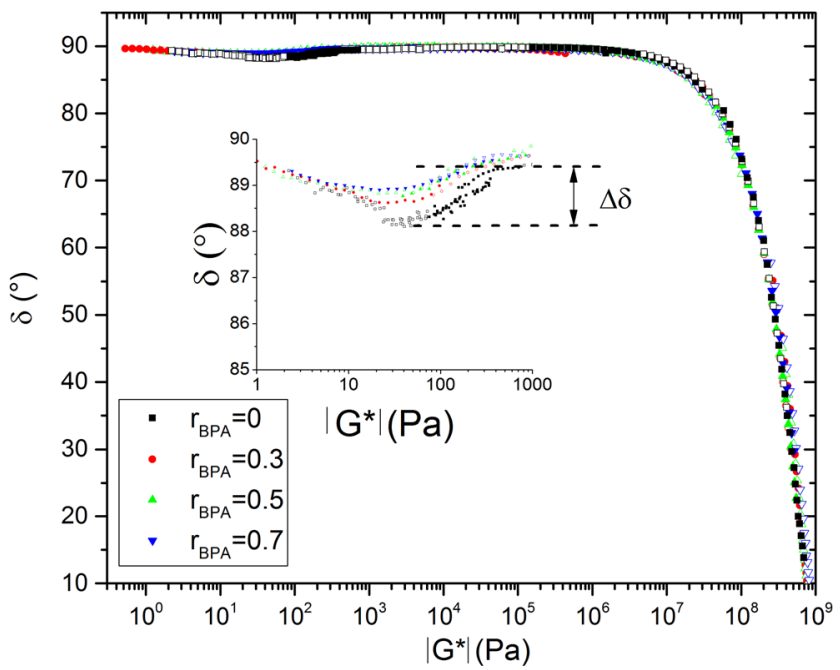

Fig. 6 Van Gurp-Palmen plots $\delta\left(\left|G^{*}\right|\right)$ for the DGEBA/BPA/SiO suspensions with various concentrations of BPA. The curves are synthesized from the data delivered by the isothermal frequency sweeps without any treatment. The inset allows a magnified view on the anomalous behaviour of the phase angle at small values of the complex moduli $\left|G^{*}\right|$

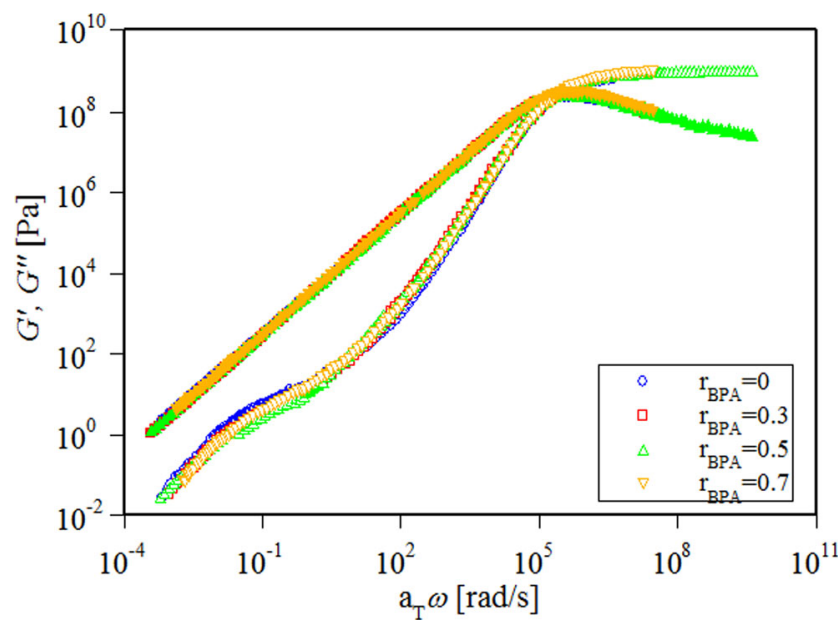

Fig. $7 G^{\prime}\left(a_{T} \omega\right)$ (open symbols) and $G^{\prime \prime}\left(a_{T} \omega\right)$ (closed symbols) from Fig. 3 shifted by a horizontal factor to the master curves of the DGEBA/SiO suspension. The corresponding normalization shift factors can be found in Table 4

a function of the volume concentration $\left(x_{\mathrm{V}}\right)$ of silica nanoparticles. In addition to $\Delta \delta\left(r_{\mathrm{BPA}}\right)$, Fig. 8 also shows the $x_{\mathrm{V}}$ dependence of the loss angle change $(\Delta \delta)$ as calculated from data published in Dannert et al. (2015). Clearly, $\Delta \delta$ increases with the filler content. The fact that the change of the loss angle at low frequencies does not scale with the molecular weight of the matrix but obviously increases with the amount of filler particles leads to the conclusion that the observed lowfrequency process is induced by the filler particles and not by an intrinsic property of the matrix. In the study of Dannert

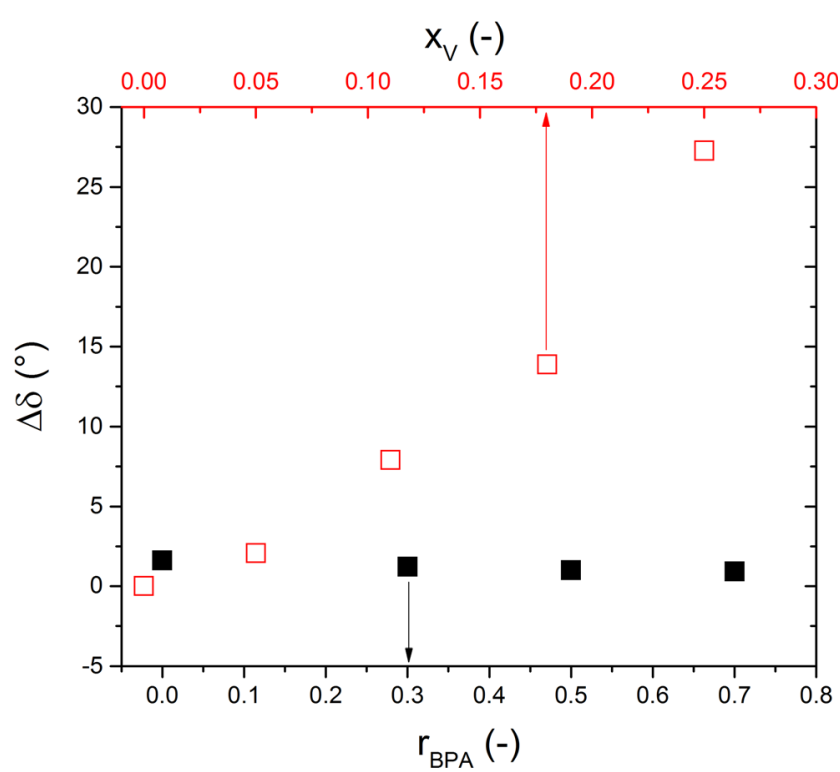

Fig. 8 Loss angle change $(\Delta \delta)$ induced by $\mathrm{SiO}_{2}$ nanoparticles in DGEBA/BPA/SiO $\mathrm{S}_{2}$ suspensions. Full squares (lower horizontal axis) $\Delta \delta$ as a function of the mass ratio $\left(r_{\mathrm{BPA}}\right)$ evaluated from the van GurpPalmen plot in Fig. 6 (filler volume concentration, $x_{\mathrm{V}}=0.05$ ). Open squares (upper horizontal axis) $\Delta \delta$ as a function of the $\mathrm{SiO}_{2}$ volume concentration $\left(x_{\mathrm{V}}\right)\left(\mathrm{BPA}\right.$ mass ratio, $\left.r_{\mathrm{BPA}}=0\right)$ obtained from Fig. 1 
et al. $(2014,2015)$, the low-frequency event has been identified as a Brownian relaxation.

\section{Influence of suspension viscosity on Brownian relaxation in suspensions}

Having established and characterized a model system formed by a series of nanoparticle suspensions which only differ in the viscosity, this system is now used to investigate the dependency of the Brownian relaxation process on suspension viscosity. For a quantitative description, the Brownian relaxation times need to be determined. This is not straightforward since the Brownian relaxation process is in competition with the $\alpha$-process of the matrix - both processes overlap on the frequency scale (see Fig. 5). A method to overcome this difficulty is described in detail in Dannert et al. (2015). The obtained relaxation times are listed in Table 5 and represented in Fig. 9 in a double-logarithmic plot as a function of the suspension viscosity $\left(\eta_{\mathrm{s}}\right)$. A linear fit of the data leads to a slope of 0.98 fortifying the expectation that Brownian relaxation times are proportional to the suspension viscosity.

Several authors state that Brownian relaxation times can be described by diffusion times

$\tau=\frac{\pi a l^{2}}{k_{\mathrm{B}} T} \eta_{\mathrm{s}}$

where $l$ represents a characteristic length scale, $k_{\mathrm{B}}$ the Boltzmann constant, $T$ the temperature, $a$ the particle radius and $\eta_{\mathrm{s}}$ the suspension viscosity (Banchio et al. 1999; Shikata and Pearson 1994; Sohn and Rajagopalan 2004; van der Werff et al. 1989; Watanabe et al. 1997). In a previous paper (Dannert et al. 2015), the authors showed that the surface-tosurface mean particle distance (Bansal and Ardell 1972)

$l=2 a\left(\left[\frac{0.63}{x_{\mathrm{V}}}\right]^{-1 / 3}-1\right)$

can be used as a characteristic length scale to adequately describe the concentration dependency of Brownian relaxation times over an extended concentration range.

Inserting Eq. (6) into Eq. (5) and setting $a=12.5 \mathrm{~nm}, T=$ $273 \mathrm{~K}$ and $x_{\mathrm{V}}=0.05$ allow to calculate the diffusion time as a function of the suspension viscosity. The result is shown in Fig. 9 (red solid line) along with the relaxation times (open

Table 5 Brownian relaxation times $(\tau)$ for the different mass ratios $\left(r_{\mathrm{BPA}}\right)$

\begin{tabular}{lll}
\hline$r_{\mathrm{BPA}}(-)$ & $\tau(\mathrm{s})$ & $\eta_{\mathrm{s}}\left(\mathrm{Pa} \mathrm{s}^{-1}\right)$ \\
\hline 0 & $4.41 \times 10^{1}$ & $2.67 \times 10^{3}$ \\
0.3 & $8.13 \times 10^{3}$ & $5.49 \times 10^{5}$ \\
0.5 & $6.05 \times 10^{5}$ & $4.78 \times 10^{7}$ \\
0.7 & $1.55 \times 10^{7}$ & $1.28 \times 10^{9}$ \\
\hline
\end{tabular}

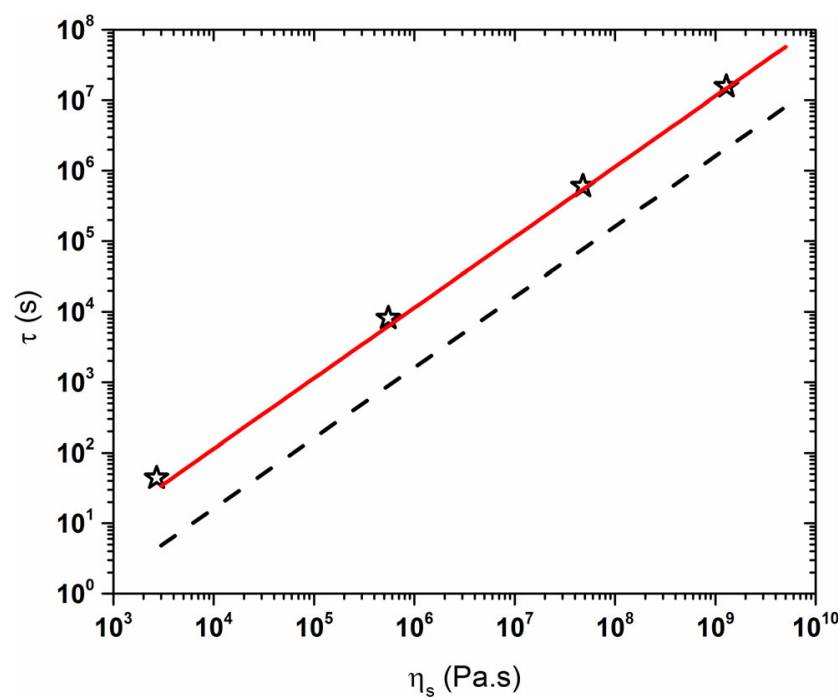

Fig. 9 Relaxation times $(\tau)$ as a function of the suspension viscosity $\left(\eta_{\mathrm{s}}\right)$. All values are taken from Table 4 . The red solid line corresponds to the diffusion times $(\tau)$ (Eq. (5)) with the surface-to-surface mean particle distance as length scale (Eq. (6)) using the parameters $a=12.5 \mathrm{~nm}, T=$ $273 \mathrm{~K}$ and $x_{\mathrm{V}}=0.05$. The dashed line illustrates the variation of the Peclet time calculated for the same parameters

stars) obtained from the experimental data as described above. Both data sets perfectly coincide, despite the fact that the diffusion times are calculated and do not originate from a fit of Eq. (5) to the experimental data. Using the classical Peclet time ( $l=a$ instead of Eq. (6)) yields the black dashed line shown in Fig. 9. Clearly, this classical expression for the diffusion time does only qualitatively but not quantitatively describe the experimental data - a result which confirms previous findings (Dannert et al. 2015).

\section{Conclusion}

In this contribution, we have presented a series of nanocomposite suspensions exhibiting two essential properties: (i) The absolute values obtained for the suspension viscosities differ by 5 orders of magnitude, and (ii) the character of the viscoelastic behaviour as depicted by the master curves of both the storage and the loss part of the elastic shear modulus is always the same, independent of the suspension viscosity. Both features make the system an ideal candidate to investigate the relation between viscosity and dynamics of Brownian relaxation in suspensions with identical particles, interactions and average interparticle distance. Since the Brownian relaxation is caused by the stress induced by the Brownian movement of particles in suspensions, a direct link between the viscosity of the medium in which the particles are moving and the timescale of their relaxation can be expected and has already been stated by phenomenological (Shikata and Pearson 1994) as well as theoretical (Banchio et al. 1999) considerations. In the present study, we could show that there exists a linear 
relation between Brownian relaxation times and suspension viscosities. Moreover, the presented data quantitatively confirms a phenomenological description (Dannert et al. 2015) which uses interparticle distances as characteristic length scales instead of the widely used particle radius.

Open Access This article is distributed under the terms of the Creative Commons Attribution 4.0 International License (http:// creativecommons.org/licenses/by/4.0/), which permits unrestricted use, distribution, and reproduction in any medium, provided you give appropriate credit to the original author(s) and the source, provide a link to the Creative Commons license, and indicate if changes were made.

\section{References}

Baller J, Becker N, Ziehmer M, Thomassey M, Zielinski B, Müller U, Sanctuary R (2009) Interactions between silica nanoparticles and an epoxy resin before and during network formation. Polymer 50: 3211-3219

Banchio AJ, Nägele G, Bergenholtz J (1999) Viscoelasticity and generalized Stokes-Einstein relations of colloidal dispersions. J Chem Phys 111:8721-8740

Bansal PP, Ardell AJ (1972) Average nearest-neighbor distances between uniformly distributed finite particles. Metallography 5:97-111

Baumgaertel M, Schausberger A, Winter HH (1990) The relaxation of polymers with linear flexible chains of uniform length. Rheol Acta 29:400-408

Capaccioli S, Corezzi S, Gallone G, Rolla P, Comez L, Fioretto D (1998) Dynamics of epoxies: a full dielectric analysis by wideband spectroscopy. J Non-Cryst Solids 235:576-579

Corezzi S, Beiner M, Huth H, Schröter K, Capaccioli S, Casalini R, Fioretto D, Donth E (2002) Two crossover regions in the dynamics of glass forming epoxy resins. J Chem Phys 117:2435-2448

Dannert R, Sanctuary R, Baller J (2015) Unexpected maximum in the concentration dependency of the relaxation frequency of Brownian stress in a colloidal suspension. J Rheol 59:391-404
Dannert R, Sanctuary R, Thomassey M, Elens P, Krüger JK, Baller J (2014) Strain-induced low-frequency relaxation in colloidal DGEBA/SiO2 suspensions. Rheol Acta 53:715-723

Debenedetti PG, Stillinger FH (2001) Supercooled liquids and the glass transition. Nature 410:259-267

Ferry JD (1980) Viscoelastic properties of polymers. John Wiley \& Sons, Hoboken

Fox TG, Flory PJ (1950) Second-order transition temperatures and related properties of polystyrene. I. Influence of molecular weight. J Appl Phys 21:581-591

Paluch M, Roland C, Gapinski J, Patkowski A (2003) Pressure and temperature dependence of structural relaxation in diglycidylether of bisphenol A. J Chem Phys 118:3177-3186

Sanctuary R, Baller J, Krüger J-K, Schaefer D, Bactavatchalou R, Wetzel B, Possart W, Alnot P (2006) Complex specific heat capacity of two nanocomposite systems. Thermochim Acta 445:111-115

Shikata T, Pearson DS (1994) Viscoelastic behavior of concentrated spherical suspensions. J Rheol 38:601-616

Smith ME, Ishida H (1994) Kinetics of the condensation reaction of epoxide with phenol: linear chain growth versus branching. Macromolecules 27:2701-2707

Sohn I, Rajagopalan R (2004) Microrheology of model quasi-hard-sphere dispersions. J Rheol 48:117-142

van der Werff J, de Kruif C, Blom C, Mellema J (1989) Linear viscoelastic behavior of dense hard-sphere dispersions. Phys Rev A 39:795807

Van Gurp M, Palmen J (1998) Time-temperature superposition for polymeric blends. Rheol Bull 67:5-8

Watanabe H, Yao M-L, Osaki K, Shikata T, Niwa H, Morishima Y (1997) Nonlinear rheology of a concentrated spherical silica suspension. Rheol Acta 36:524-533

White KL (2013) Rheology of model nanoparticle suspensions in epoxy. Dissertation, Texas A\&M University

Winter HH (2013) Glass transition as the rheological inverse of gelation. Macromolecules 46:2425-2432

Winter HH, Mours M (2006) The cyber infrastructure initiative for rheology. Rheol Acta 45:331-338 